\title{
El tejo en el sur de España: análisis geoecológico y propuesta de conservación de una población mediterránea en peligro crítico de extinción
}

\author{
The yew in southern Spain: geo-ecological analysis and proposal of conservation of a critically \\ endangered Mediterranean population
}

\author{
José Antonio Olmedo-Cobo *, José Gómez-Zotano \\ * Autor de Correspondencia: Universidad de Granada, Departamento de Análisis Geográfico Regional y Geografía Física, \\ Campus de Cartuja s/n, Granada, España, tel.: 0034-626845825, jaolmed@correo.ugr.es
}

\begin{abstract}
SUMMARY
This paper introduces a geo-ecological analysis done on a relict, threatened and little known Mediterranean population of yew (Taxus baccata). We study the case of Sierra Arana (southern Spain). The priority is to know under what conditions the species develops and its ability for future preservation, for which, through fieldwork -the main methodological procedure-, its identification and location and the analysis of not only its population demographic structure, but also of its conservation status, its habitats and ecological niches are undertaken. The results obtained are a chorological map of the species and a major knowledge of it; establishing: a) predominance of mature trees -middle-aged and long-lived-, scarcity of young specimens and absence of seedlings; b) a significant imbalance in sex ratio, prevailing male individuals; c) a conservation status that varies from optimum to degraded for the population as a whole; d) habitats located on very steep slopes over basic soils, even in sub-rupicolous locations; e) ecological niche corresponds to preforestal orla of deciduous and marcescent forests of Acer monspessulanum and Quercus faginea. Finally, we present a proposal for the conservation and regeneration of yew based on the protection of existing specimens and on the reforestation in the most appropriate ecological niches. The main conclusion established is the critically endangered state of Taxus baccata in Sierra Arana due to its little chance of natural regeneration and expansion, requiring extraordinary measures to its preservation.
\end{abstract}

Key words: Taxus baccata, Sierra Arana, southern end distribution, relict, reforestation.

\section{RESUMEN}

Este trabajo consiste en un análisis geoecológico de una población mediterránea relicta, amenazada y poco conocida de tejo (Taxus baccata). Se estudia el caso de Sierra Arana (sur de España). El objetivo prioritario es conocer en qué condiciones se desarrolla la especie y sus posibilidades de preservación en el futuro, para lo cual se aborda, mediante el trabajo de campo -principal procedimiento metodológico-, su identificación y localización, el análisis de la estructura demográfica de la población, de su estado de conservación, de los hábitats y de los nichos ecológicos. Como resultados se obtienen un mapa corológico de la especie y un mayor conocimiento de la misma, estableciéndose: a) el predominio de árboles adultos de edades medias y longevas, la escasez de individuos juveniles y la inexistencia de plántulas; b) un destacado desequilibrio sexual, con prevalencia del género masculino; c) un estado de conservación óptimo a degradado para el conjunto de la población; d) unos hábitats en laderas acusadas sobre suelos básicos, en emplazamientos incluso subrupícolas; e) un nicho ecológico coincidente con la orla preforestal de bosques caduco-marcescentes de Acer monspessulanum y Quercus faginea. También se plantea una propuesta de conservación y regeneración del tejo basada en la protección de los ejemplares existentes y en la reforestación de los nichos ecológicos más adecuados. Se concluye que Taxus baccata en Sierra Arana se encuentra en peligro crítico de extinción a consecuencia de sus escasas posibilidades de regeneración y expansión de manera natural, requiriendo de medidas extraordinarias para su conservación.

Palabras clave: Taxus baccata, Sierra Arana, distribución finícola meridional, carácter relicto, reforestación.

\section{INTRODUCCIÓN}

El tejo, Taxus baccata L., presenta una amplia distribución geográfica en Europa. Como único representante de la familia Taxaceae, alcanza las penínsulas escandinava, anatólica e ibérica (Vidakovic 1991, Thomas y Polwart 2003). También aparece localmente en el Norte de África -cordilleras del Rif y del Atlas- y testimonialmente en los archipiélagos macaronésicos de Azores y Madeira (Tho- mas y Polwart 2003, Charco 2006). Sin embargo, las poblaciones que componen esta extensa área corológica se caracterizan por un notable grado de fragmentación y, por lo general, por un bajo número de individuos. Esto es debido a la progresiva degradación de sus nichos ecológicos, tanto por la mayor templanza y sequedad climáticas tras el último máximo glacial en Europa, como por la intensa presión antrópica a la que se ha sometido a la especie y sus hábitats forestales desde el Neolítico reciente a la actualidad, 
siendo uno de los árboles más afectados por la intervención humana (Svenning y Magard 1999). Bajo estas circunstancias, la regresión de las poblaciones de $T$. baccata ha sido especialmente notable en la Cuenca Mediterránea: el detrimento de los bosques húmedos eurosiberianos (hayedos, robledales y abetales) - de los que el tejo comúnmente forma parte-, ha ido acompañado de una importante expansión de las formaciones esclerófilas (Ruiz-Labourdette et al. 2012). En la actualidad, el déficit de agua es el factor que dirige su extensión y distribución geográfica en este territorio (Sanz et al. 2009), limitándose a ambientes húmedos en cotas progresivamente más elevadas de las principales cordilleras (Thomas y Polwart 2003, García et al. 2000). En estos enclaves se conservan teselas relictas de tejedas eurosiberianas que ocupan nichos ecológicos herencia de los de sus ancestros (Nogués-Bravo 2009).

En la Península Ibérica, la Cordillera Bética constituye uno de los núcleos mediterráneos europeos donde T. baccata se encuentra más amenazado en relación con su distribución finícola meridional; en algunos de sus enclaves se considera un elemento holártico relicto en peligro inminente de extinción (Cabezudo et al. 2011). En este contexto biogeográfico, la especie forma parte de comunidades de gimnospermas que ocupan posiciones ecológicas marginales respecto a las formaciones climácicas dominadas por fagáceas (Quercus rotundifolia Lam., Q. suber L., Q. faginea, Q. canariensis Willd. y Q. pyrenaica Willd) (Pérez-Latorre et al. 1999). Si bien estas formaciones de gimnospermas todavía presentan una amplia, aunque muy discontinua distribución en estas montañas (Valle et al. 1988, Regato et al. 1995, Torres et al. 1996), su dinámica y evolución reciente es regresiva, no sólo por razones climáticas, sino también, y sobre todo, por una masiva y secular deforestación. En este contexto geohistórico $T$. baccata ha encontrado refugio en enclaves poco accesibles, en parte hábitats subrupícolas, y siempre en emplazamientos con orientación Norte o cerca de arroyos de montaña donde puede soportar las adversas condiciones estivales mediterráneas.

Aunque el conocimiento actual de las tejedas béticas es pródigo, todavía existen algunas poblaciones poco conocidas, bien por su escaso número de individuos, por lo que pasan desapercibidas en el conjunto del paisaje vegetal, bien por localizarse en emplazamientos de media-alta montaña inaccesibles. Así sucede con la tejeda existente en Sierra Arana, donde se conservan unas decenas de pies a altitudes comprendidas entre 1.600 y 1.850 m s.n.m. La presencia de la especie en el macizo ha sido citada por distintos autores y queda reflejada en el Mapa Forestal de España escala 1:200.000 (MAPA 1991), aunque hasta el momento no se ha abordado un estudio exhaustivo de su significación ecológica.

Este artículo pretende solventar dicho desconocimiento a partir del análisis geoecológico de T. baccata en Sierra Arana. Se parte de una serie de hipótesis iniciales que sustentan la investigación: a) el degradado estado de conservación de la especie en el macizo apunta, a priori, a una situación cercana a su extinción y, en consecuencia, requiere de su consideración como elemento relicto amenazado de alta singularidad; b) las condiciones ecológicas de los microhábitats donde se refugia y las características demográficas de la población dificultan su regeneración y expansión de manera natural; c) la recuperación de T. baccata sería posible mediante el establecimiento de una estrategia adecuada para su conservación basada en la protección de sus nichos ecológicos y, especialmente, en la reforestación. Para dar respuesta a estas hipótesis, se cubre una serie de objetivos: (i) la localización de la totalidad de los tejos existentes en Sierra Arana, y la representación cartográfica de su areal corológico actual; (ii) el estudio de la estructura demográfica y del estado de conservación de los distintos núcleos que integran la población; (iii) la caracterización del tipo de hábitats; (iv) la identificación de los nichos ecológicos donde se desarrolla la especie; (v) la propuesta de una serie de actuaciones encaminadas a la regeneración y protección de $T$. baccata y sus nichos ecológicos. Esta información constituye una herramienta valiosa para la gestión de la especie de cara a su preservación en otros ámbitos del Sur de España donde se encuentra, igualmente, en peligro de extinción.

\section{MÉTODOS}

Área de estudio. Sierra Arana $\left(37^{\circ} 24^{\prime}-37^{\circ} 16^{\prime} \mathrm{N} ; 3^{\circ} 16^{\prime}\right.$ - 3035' O; Peña de la Cruz, 2.027 m s.n.m.) abarca una extensión de $175 \mathrm{~km}^{2}$, ocupando una posición central en la Cordillera Bética (figura 1). Es un macizo relativamente aislado y rodeado de tierras llanas por el Oeste, Este y Norte; el relieve montañoso sólo tiene continuidad hacia el Sur por las primeras estribaciones de Sierra Nevada. Esta configuración física le otorga un carácter de isla climática y biogeográfica, que lo convierte en un centro destacado de especiación de flora y fitodiversidad, valores que, junto a otros de tipo faunístico y geomorfológico, ha propiciado su inclusión en la Red Natura 2000 ("Lugar de Interés Comunitario" -LIC-y "Zona de Especial Protección para las Aves" -ZEPA-) y, parcialmente, en el Parque Natural de la Sierra de Huétor.

De carácter estructural, el macizo forma parte de las Zonas Externas de la Cordillera Bética. Su litología es básicamente calizo-dolomítica, lo que favorece un modelado kárstico formado por dolinas, lapiaces, microlapiaces, mesas de travertinos, cañones fluvio-kársticos, simas y cuevas. Los suelos son mayoritariamente litosoles calcáreos escasamente evolucionados, aunque más desarrollados en zonas de vaguada o en depresiones de disolución kárstica.

El clima es genuinamente mediterráneo, aunque con un destacado matiz de montaña donde la altitud modula los regímenes térmico y pluviométrico a la baja y al alza respectivamente. En la zona basal, la temperatura media anual es de $13,5^{\circ} \mathrm{C}$ y la pluviometría de $710 \mathrm{~mm}$ por año (Huétor-Santillán, $1.060 \mathrm{~m}$ s.n.m., datos propios). En la zona de cumbres se estimó un valor promedio de $6{ }^{\circ} \mathrm{C}$ a 


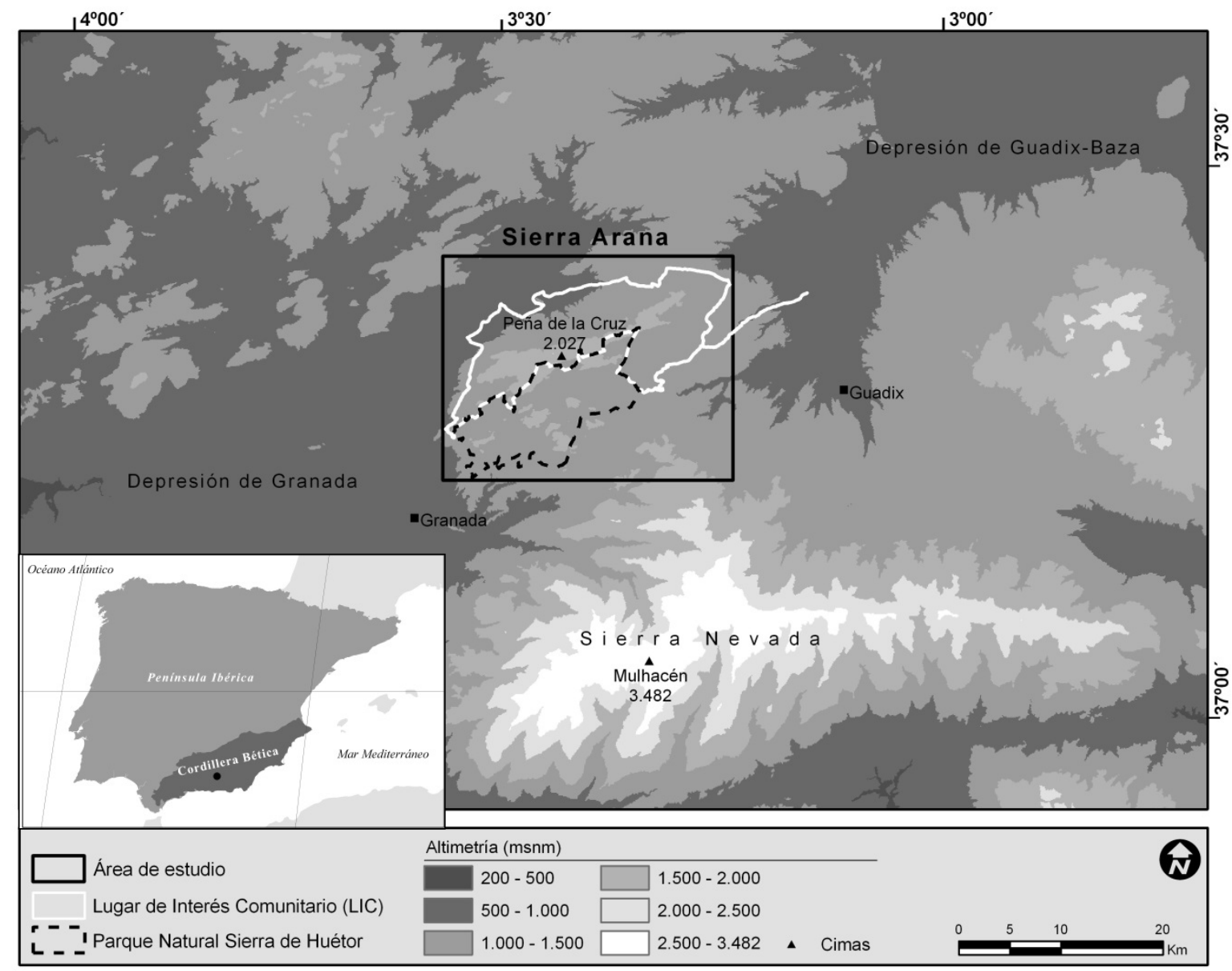

Figura 1. Localización de Sierra Arana en los contextos geográficos de la Península Ibérica y Cordillera Bética (arriba) y en el marco regional (abajo).

Location of Sierra Arana in the geographical contexts of the Iberian Peninsula and Baetic Mountain Range (top) and in the regional context (below).

partir de lo establecido por Olmedo-Cobo (2011) para la cercana Sierra de Baza; las precipitaciones anuales son superiores a $1.000 \mathrm{~mm}$ (a $1.400 \mathrm{~m}$ s.n.m. se recogen $900 \mathrm{~mm}$ por año, datos propios), con un destacable gradiente pluviométrico positivo por retención orográfica en las vertientes orientadas al Oeste; el régimen de precipitaciones presenta un máximo otoño-invernal que decae hacia la primavera, destacando la casi total ausencia de lluvias en el trimestre estival $(<50 \mathrm{~mm}$ entre mediados de junio y mediados de septiembre).

En cuanto al bioclima, se considera el tipo pluviestacional-oceánico para el conjunto de Sierra Arana. Dentro de los umbrales bioclimáticos establecidos para la región Mediterránea por Rivas-Martínez y Loidi (1999) y Rivas-Martínez (2008), el macizo queda comprendido en su mayor parte en el piso bioclimático supramediterráneo subhúmedo (Itc ${ }^{1}$ :

Itc (índice de termicidad compensado): Itc: It $\pm \mathrm{C}$, donde It [índice de termicidad, $(\mathrm{T}+\mathrm{m}+\mathrm{M}) 10$ ] es la suma (décimas de ${ }^{\circ} \mathrm{C}$ ) de la temperatura media anual $(\mathrm{T})$ y las temperaturas medias de mínimas $(\mathrm{m})$ y máximas $(\mathrm{M})$ del mes más frío; $\mathrm{C}$ es un valor de compensación para las zonas extratropicales de la Tierra (Rivas-Martínez 2008).
210 - 80; Io²: 3,6 - 6); la zona basal presenta condiciones mesomediterráneas (Itc: 210 - 350) seco-subhúmedas (Io: $2-6)$ y las cumbres más elevadas se consideran oromediterráneas subhúmedas (Itc: 80 - -93; Io: 3,6-6). Estos umbrales se concretan físicamente mediante el reconocimiento de especies fitoindicadoras.

De acuerdo con la última revisión de la sectorización biogeográfica de este territorio llevada a cabo por RivasMartínez (2011), Sierra Arana y los territorios montañosos adyacentes (sierras de Huétor, de la Alfaguara y cuenca del río Aguas Blancas) constituyen, fruto de su fuerte personalidad biogeográfica, el distrito Alfacarino del sector biogeográfico Granadino-Almijarense (subsector Granadino), perteneciente a la provincia Bética (región Mediterránea, reino Holártico).

Considerando los estudios sobre sintaxonomía, series de vegetación y asociaciones vegetales de Rivas-Martínez

\footnotetext{
Io (índice ombrotérmico): 10P/T, donde $\mathrm{P}$ es la suma de la precipitación media (mm) de los meses con temperatura media $>0{ }^{\circ} \mathrm{C}$, y T es la suma (décimas de ${ }^{\circ} \mathrm{C}$ ) de las temperaturas medias de los meses con valores positivos (Rivas-Martínez 2008).
} 
et al. (1997), Valle (2003) y Rivas-Martínez (2008, 2011), en la actualidad T. baccata en Sierra Arana forma parte de un dominio forestal potencial de bosque caduco-marcescente de carácter húmedo y de su orla de prebosque. Este bosque integra la asociación vegetal Daphno latifoliaeAceretum granatensis Rivas-Martínez 1965, que constituye el clímax de la serie de vegetación del mismo nombre, Daphno latifoliae-Aceretum granatensis Sigmetum, definida como temporihigrófila, bética, calcícola, supramediterránea subhúmedo-húmeda de los bosques de Acer opalus Mill. ssp. granatense (Boiss.) Font Quer y Rothm. con Daphne laureola (Rivas-Martínez 2011). La figura 2 representa la geoserie de vegetación potencial donde se inserta la serie Daphno-Aceretum granatensis $S$.

Proceso metodológico. La consecución de los objetivos que integran el análisis geoecológico planteado requirió del trabajo de campo, principal procedimiento metodológico de la investigación, dada la ausencia de fuentes bibliográficas de referencia; para la identificación y localización de T. baccata y de los micro-ambientes donde se refugia, se estableció una serie de itinerarios que permitieron el barrido sistemático del área de estudio, el conteo y la geo-referenciación de los ejemplares existentes. Esta fase del método conllevó la realización de encuestas orales sobre los usos y aprovechamientos tradicionales del tejo.

Para el análisis de la estructura demográfica de T. baccata se procedió a la diferenciación por género de los in- dividuos mediante el reconocimiento, en campo, de los arilos femeninos en otoño y de los conos masculinos en primavera. También se estableció la razón de sexo de cada núcleo (\% del número de individuos femeninos -productores de semillas- respecto al total de ejemplares). La estimación de la edad del arbolado se fue realizada a partir de las mediciones del diámetro basal del tronco, teniendo en cuenta los parámetros de crecimiento medio anual establecidos por Hulme (1996) (1 mm por año) y Burgarella et al. (2010) (2,86 mm por año).

Para la caracterización de los tipos de hábitats se realizó un estudio mesológico con especial atención a los parámetros litología, pendiente, relieve, suelos, altitud y orientación. Para el establecimiento del tipo de roquedo y suelo se empleó el Mapa Geológico de España (1:50.000), los Mapas de Suelos del Proyecto Lucdeme (1:100.000) y el Mapa Digital de Suelos de la provincia de Granada de Oyonarte et al (2006).

Para la identificación de los nichos ecológicos se utilizó una metodología biogeográfica y fitosociológica integrada consistente en el análisis del paisaje vegetal, el bioclima y la biogeografía (Alcaraz 1996). En el reconocimiento y comprobación de la vegetación y flora se consultaron las obras de Valle (2003), Castroviejo (2010) y Blanca et al. (2011) como fuentes bibliográficas de apoyo.

El trabajo de gabinete consistió, fundamentalmente, en la elaboración de la cartografía que se incorporó al análisis geoecológico. Se empleó SIG (sistemas de información geográfica) mediante el programa ArcGis 9.3.

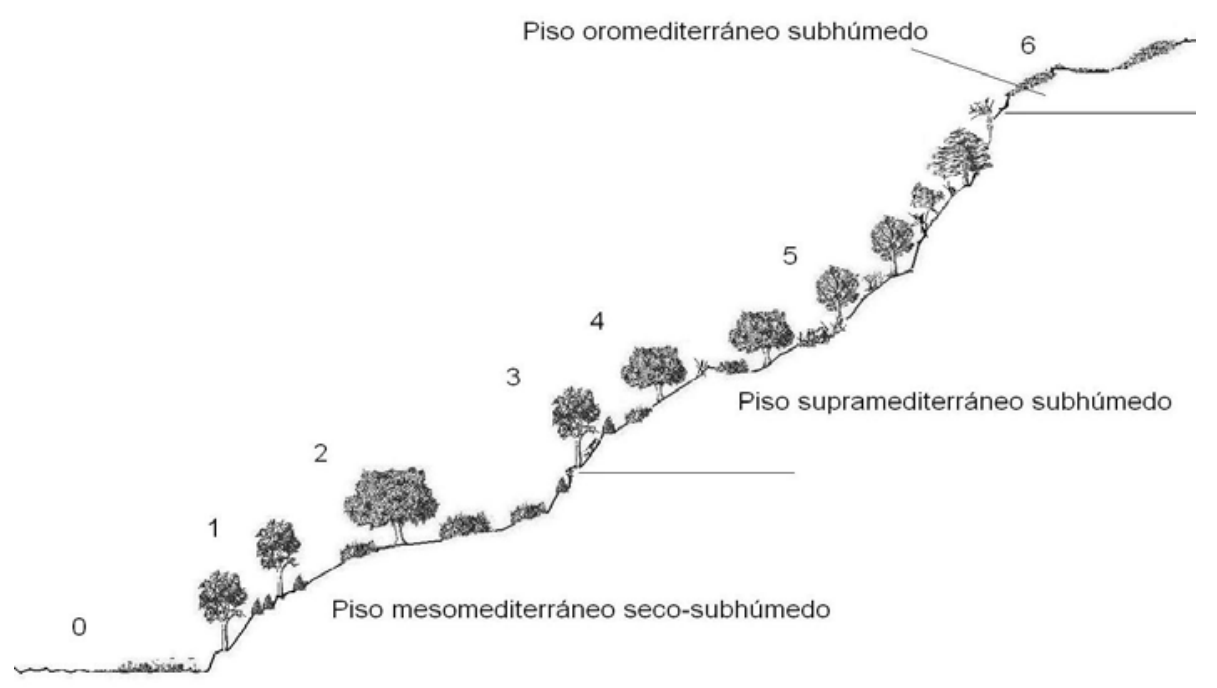

Figura 2. Geoserie de vegetación potencial en Sierra Arana.

0, Eriales y cultivos (dominio potencial de 2). 1, Rhamno myrtifolii-Juniperetum phoeniceae S. 2, Paeonio coriaceae-Quercetum rotundifoliae S. 3 , Rhamno myrtifolii-Juniperetum phoeniceae S. 4, Berberido hispanicae-Quercetum rotundifoliae S. 5, Daphno latifoliae-Aceretum granatensis S. 6, Junipero sabinae-Pinetum latisquamae $S$. Geoserie of potential vegetation in Sierra Arana.

0, Wastelands and fields of crops (potential domain of 2). 1, Rhamno myrtifolii-Juniperetum phoeniceae S. 2, Paeonio coriaceae-Quercetum rotundifoliae S. 3, Rhamno myrtifolii-Juniperetum phoeniceae S. 4, Berberido hispanicae-Quercetum rotundifoliae S. 5, Daphno latifoliae-Aceretum granatensis S. 6, Junipero sabinae-Pinetum latisquamae $S$. 
Para el establecimiento de una estrategia encaminada a la conservación y regeneración de T. baccata en Sierra Arana, se analizaron aquellas experiencias exitosas desarrolladas en otros contextos geográficos béticos de similares características ecológicas, y recogidas por Costa (2006).

\section{RESULTADOS}

Caracterización geoecológica de T. baccata en Sierra Arana. Identificación y localización espacial. Se han localizado tres núcleos (P) de T. baccata en Sierra Arana que totalizan 24 ejemplares, todos ellos ubicados en el Suroeste del macizo a altitudes comprendidas entre 1.600 y 1.850 m s.n.m., es decir, dentro del piso supramediterráneo típico y superior, bajo ombroclima subhúmedo (cuadro 1 y figura 3): P1, Paraje del "Cerro de los Tejos", con una superficie aproximada de tres hectáreas, es el principal tanto por el número de individuos que lo componen -16- como por el estado de conservación que presentan los árboles; P2, Paraje de "Altos de Majalijar", presenta una extensión de 10 hectáreas con siete ejemplares; y P3, Paraje de "Río Blanco", con un área muy limitada, establecida en $100 \mathrm{~m}^{2}$, donde sólo se ha localizado un pie. Por último, hay que señalar que el núcleo P1 y, parcialmente el P2, quedan dentro del área de protección del Parque Natural de la Sierra de Huétor.

Estructura demográfica y estado de conservación. El núcleo P1 (figura 4), formado por 16 ejemplares, presenta 10 árboles adultos o de edad media y seis individuos juveniles, aunque en ningún caso se trata de plántulas (cuadro 2). A los ejemplares más longevos, cuya talla oscila entre 4 y $8 \mathrm{~m}$ de altura, se les atribuye un rango de edad aproximada de 118 a 230 años según la razón de crecimiento establecida por Burgarella et al. (2010), o bien de 340 a 660 años según la tasa de incremento diametral señalada por Hulme (1996), correspondientes con unos diámetros basales de tronco de entre 0,34 y 0,66 m. La complicada estructura de los troncos en algunos individuos, muy ramificada a pocos centímetros por encima del suelo, dificulta en determinados casos, la medida exacta de los diámetros (lo que también sucede en P2). Los individuos más jóvenes tienen portes de 1,5 a 3,5 m, con diámetros de tronco de 0,05 a 0,1 $\mathrm{m}$, lo que les otorga edades aproximadas de 19 a 35 años (2,86 mm por año) o de 55 a 100 años (1 mm por año).
Se logró identificar el sexo de todos los ejemplares de este núcleo excepto para un individuo juvenil cuyo denigrado estado lo ha impedido. Una razón de sexo de 13,3 \% denota el elevado desequilibrio existente entre individuos femeninos (2) y masculinos (13).

De los siete ejemplares que componen la población P2, 4 eran árboles adultos. Los restantes eran individuos relativamente jóvenes, aunque no de pocos años y, al igual que en el caso anterior, no se observó plántula alguna. Los primeros presentaron tallas de 5 a $9 \mathrm{~m}$, con diámetros basales de tronco de entre 0,26 y 0,64 m, por tanto con edades estimadas comprendidas entre 91 y 237 años y 260 y 640 años, respectivamente, según los dos ratios de crecimiento considerados. Los más jóvenes, por su parte, alcanzaron 2,5-3 m de altura, con diámetros de tronco de 0,08 a 0,11 $\mathrm{m}$, es decir, su longevidad fue inferior a 40 años $(2,86 \mathrm{~mm}$ por año) o en torno a los 80-110 años (1 mm por año). En este caso, existió mayor equilibrio entre géneros, siendo la razón de sexo de $50 \%$, con tres árboles femeninos, incluyendo dos de los ejemplares de mayor porte, y tres masculinos. Hubo uno más muy degradado cuyo sexo no fue posible establecer.

El único ejemplar que compone el núcleo P3 es un árbol masculino adulto, de $7 \mathrm{~m}$ de altura y un diámetro de tronco de 0,56 m, con una edad aproximada, por tanto, de entre 196 y 565 años; en este caso, la razón sexual fue 0 .

El estado de conservación de los ejemplares de $T$. baccata identificados es variable. El ramoneo del ramaje inferior de los árboles por la herbivoría del ganado vacuno -sobre todo- y de cabra montesa (Capra pyrenaica Schinz) es, en mayor o menor grado, generalizado. Resulta especialmente acusado en algunos ejemplares de P1 y P2, sobre todo en los situados hacia la base de ambos núcleos, los más accesibles a los animales; dos de los individuos localizados en P1 y P2 estaban tan dañados que no parece que puedan sobrevivir a corto-medio plazo. Por el contrario, los árboles más adultos, protegidos por un entramado vegetal denso o por su situación topográfica, gozan de un estado de conservación aceptable, destacando uno de los pies de menor edad de P1, cuya salud es excelente al crecer protegido en el seno de un rodal de espinal de alta densidad; este estrato espinoso en el resto de los casos es más abierto o incluso nulo en los emplazamientos más rocosos. La degradación zoógena afectó parcialmente al individuo

Cuadro 1. Datos geográficos de los núcleos poblacionales de Taxus baccata P1, P2 y P3.

Geographical data of the population cores of Taxus baccata P1, P2 and P3.

\begin{tabular}{ccccccc}
\hline Núcleo & $\begin{array}{c}\text { Superficie } \\
\text { (ha) }\end{array}$ & $\begin{array}{c}\text { Altitud } \\
\text { (m s.n.m.) }\end{array}$ & UTM 10x10 & N $^{\text {Individuos }}$ & Término Municipal & Parque Natural \\
\hline P 1 & 3 & $1.650-1.700$ & 30 SVG53 & 16 & Huétor Santillán & Sí \\
P 2 & 10 & $1.720-1.840$ & 30SVG52 & 7 & $\begin{array}{c}\text { Huétor Santillán } \\
\text { Cogollos de la Vega }\end{array}$ Parcial \\
P 3 & 0,01 & 1.640 & 30 SVG53 & 1 & Cogollos de la Vega & No \\
\hline
\end{tabular}




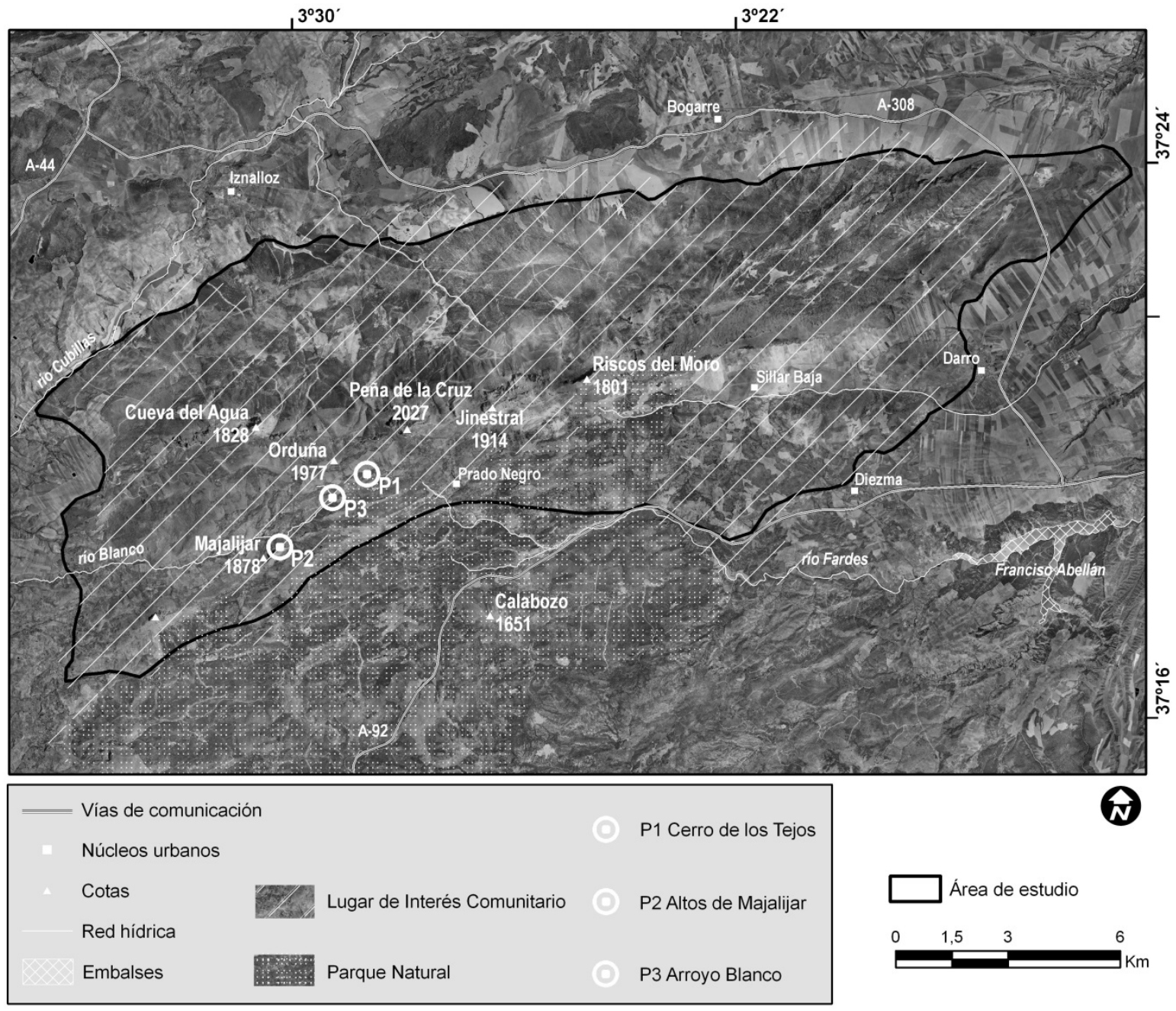

Figura 3. Localización espacial de los núcleos poblacionales de Taxus baccata P1, P2 y P3 en el contexto geográfico de Sierra Arana. Spatial location of the populations cores of Taxus baccata P1, P2 and P3 in the geographical context of Sierra Arana.

de P3, pudiéndose considerar que su estado de conservación era óptimo.

La acción perniciosa de los herbívoros se concreta, además de, en el consumo del ramaje -a pesar de su toxicidad-, en el uso de los troncos como "rascadores" por las vacunos, y el pisoteo y la compactación del suelo en el entorno inmediato de los ejemplares. Estas circunstancias son especialmente lesivas al provocar daños en la corteza protectora del tronco y una intensa erosión del suelo que hace aflorar las raíces más superficiales, que son deterioradas e incluso fragmentadas por el paso recurrente de los animales más pesados.

Tipos de hábitats. Los tres núcleos identificados se sitúan en enclaves que, debido a la conjunción de topografía, exposición-orientación y altitud, presentaban unas particulares condiciones microclimáticas marcadas por temperaturas estivales atemperadas, índices de humedad ambiental elevados buena parte del año y, como consecuencia de los notables registros pluviométricos, reservas de humedad edáfica estival suficientes para afrontar la sequedad del periodo junioseptiembre. Ello permite el desarrollo de micro-ambientes favorables para el refugio de $T$. baccata dentro del adverso contexto macro y mesoclimático conocido.

Las localidades P1 y P2 ocupan laderas de pendientes medias o acusadas en orientación Norte, siendo la inclinación progresivamente mayor al ascender en altitud. La componente edáfica se caracteriza por el desarrollo de litosoles calcáricos y regosoles litosólicos que, solo fruto de la disolución de los carbonatos, se transforman en cambisoles cálcicos más evolucionados. Los ejemplares de T. baccata situados en cotas más elevadas se enraízan directamente en paredones rocosos prácticamente verticales (figura 5) o en micro-lapiaces, donde aprovechan pequeñas grietas y fisuras del roquedo, circunstancia que refleja el carácter subrupícola y colonizador de la especie. Las laderas donde se asientan P1 y P2 culminan respectivamente a $1.718 \mathrm{~m}$ s.n.m. en la parte centro-oriental de la arista cimera del 


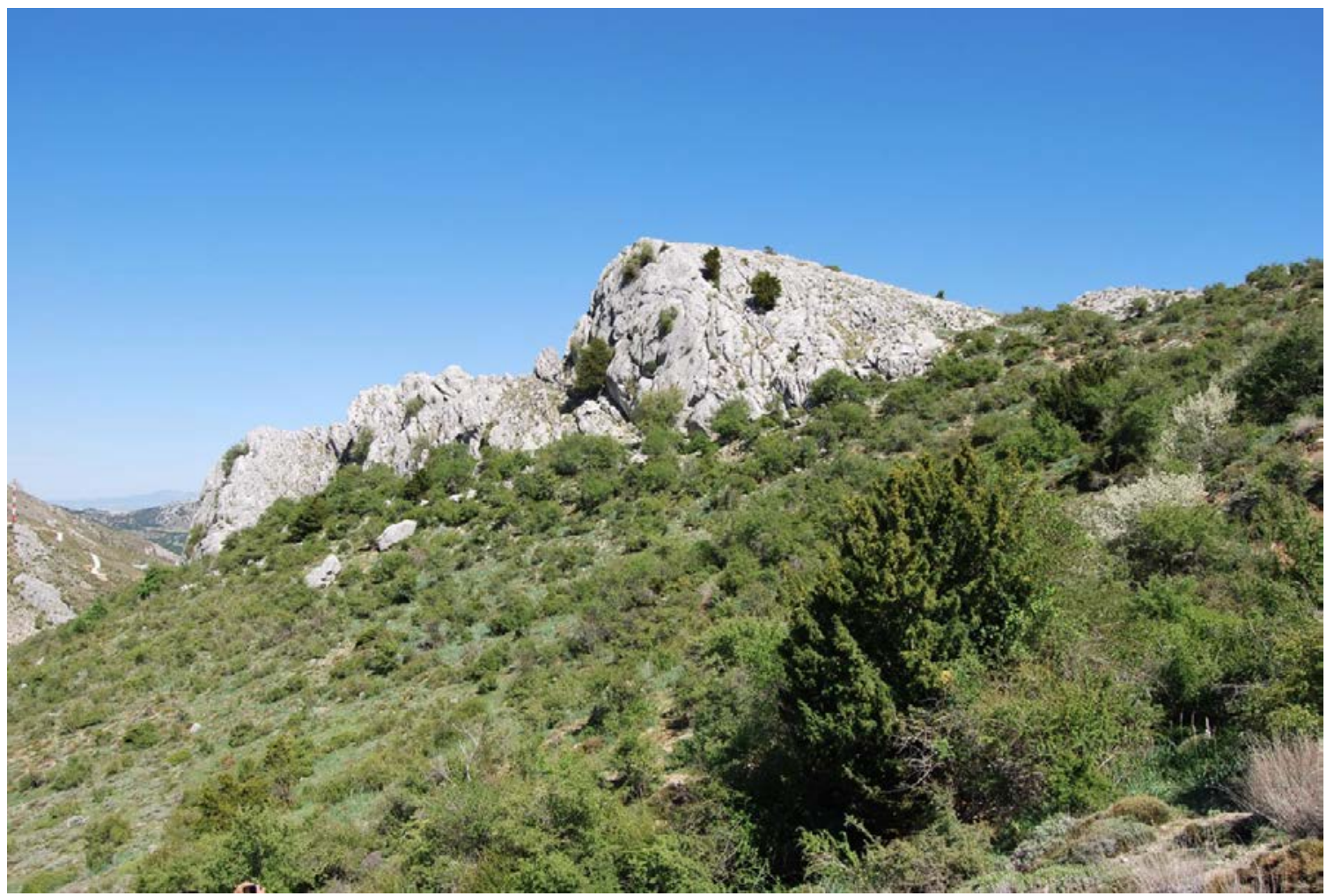

Figura 4. Visión parcial del núcleo P1, "Cerro de los Tejos”. Partial vision of core P1, "Cerro de los Tejos".

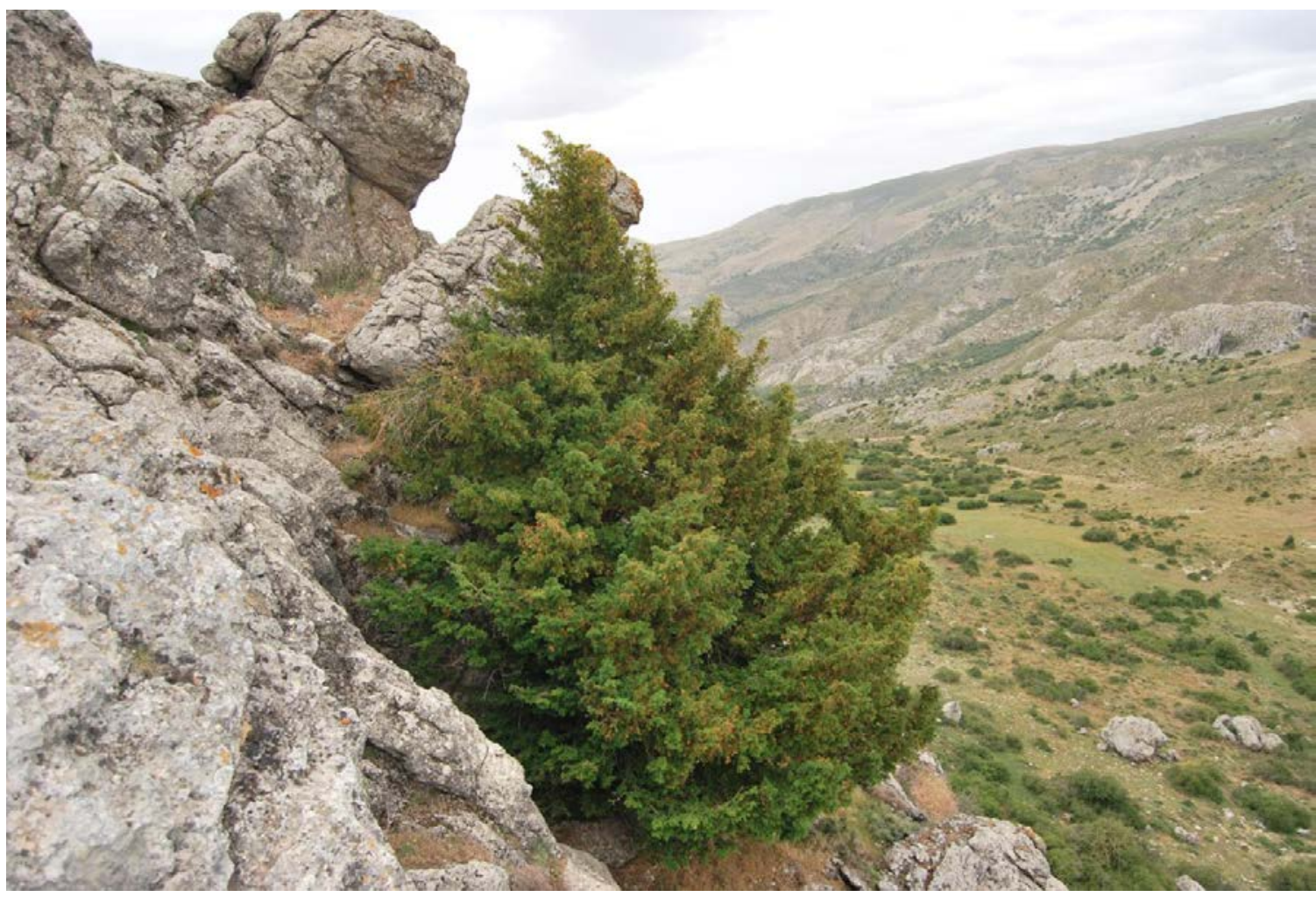

Figura 5. Ejemplar de Taxus baccata en P2, “Altos de Majalijar”, enraizado en litosoles sobre laderas de fuertes pendientes. Example of Taxus baccata in P2, "Altos de Majalijar”, which is rooted in lithosols over very steep slopes. 
Cuadro 2. Estructura demográfica y estado de conservación de Taxus baccata en P1, P2 y P3. Demographic structure and conservation status of Taxus baccata in P1, P2 and P3.

\begin{tabular}{lccccc}
\hline Núcleo-Individuo & $\begin{array}{c}\text { Talla } \\
(\mathrm{m})\end{array}$ & $\begin{array}{c}\text { Diámetro tronco } \\
(\mathrm{m})\end{array}$ & $\begin{array}{c}\text { Edad estimada } \\
(\text { años })\end{array}$ & Sexo & $\begin{array}{c}\text { Estado de } \\
\text { conservación }\end{array}$ \\
\hline P 1-1 & 5 & 0,38 & $135-385$ & Masculino & Óptimo \\
P 1-2 & 3 & 0,1 & $35-100$ & Masculino & Regular \\
P 1-3 & 1,5 & 0,07 & $26-75$ & Indeterminado & Malo \\
P 1-4 & 6 & 0,42 & $147-420$ & Masculino & Óptimo \\
P 1-5 & 2 & 0,05 & $19-55$ & Masculino & Óptimo \\
P 1-6 & 8 & 0,66 & $230-660$ & Masculino & Regular \\
P 1-7 & 4,5 & 0,49 & $173-495$ & Femenino & Óptimo \\
P 1-8 & 2,8 & 0,08 & $29-85$ & Femenino & Regular \\
P 1-9 & 8 & 0,61 & $213-610$ & Masculino & Regular \\
P 1-10 & 6 & 0,47 & $166-475$ & Masculino & Óptimo \\
P 1-11 & 5 & 0,38 & $134-385$ & Masculino & Óptimo \\
P 1-12 & 1,8 & 0,07 & $26-75$ & Masculino & Regular \\
P 1-13 & 4,5 & 0,34 & $118-340$ & Masculino & Regular \\
P 1-14 & 2 & 0,09 & $31-90$ & Masculino & Regular \\
P 1-15 & 5 & 0,37 & $129-370$ & Masculino & Regular \\
P 1-16 & 5 & $154-440$ & Masculino & Regular \\
P 2-1 & 5 & $91-260$ & Masculino & Óptimo \\
P 2-2 & 5 & $237-640$ & Masculino & Óptimo \\
P 2-3 & 9,26 & $38-110$ & Masculino & Regular \\
P 2-4 & 2,5 & $28-80$ & Indeterminado & Malo \\
P 2-5 & 2 & 0,64 & Femenino & Óptimo \\
P 2-6 & 7 & 0,11 & Femenino & Óptimo \\
P 2-7 & 0,08 & Femenino & Regular \\
P 3-1 & 0,57 & 0,61 & Masculino & Óptimo \\
\hline
\end{tabular}

Cerro de los Tejos (1.838 m s.n.m.), y a 1.878 m s.n.m. en la cumbre del pico Majalijar. Se trata, en ambos casos, de umbrías muy protegidas y poco expuestas tanto a la insolación como al embate directo de los vientos dominantes -de Oeste-Suroeste-, aunque sí abiertas a las rigurosas, aunque irregulares, "nortadas" invernales.

P3 es una localidad en orientación Sur, con escasa pendiente en el fondo de un pequeño valle que se desarrolla en dirección Norte-Sur, entre los cerros de los Tejos y de las Víboras (1.824 m s.n.m.). Es un enclave más abierto que los anteriores, si bien queda adecuadamente protegido hacia el Oeste por el Cerro de las Víboras y por su propia topografía deprimida. El efecto fondo de valle, sin embargo, favorece fenómenos de inversión térmica, desencadenantes, a su vez, de intensas y atemporales heladas primaverales que resultan negativas para $T$. baccata; frente a este hecho, el cercano nivel freático del arroyo Blanco supone una notable corrección de la desecación asociada a la orientación Sur de este enclave. El único ejemplar de T. baccata de la localidad se asienta sobre una estrecha franja de cambisol cálcico en contacto directo con los litosoles y arenosoles dolomíticos de las laderas inmediatas.

Nichos ecológicos. En las tres localidades, el nicho ecológico, es decir, la función concreta que desempeña $T$. baccata dentro del ecosistema vegetal en el que se inserta, es, si no idéntico, muy similar. La especie forma parte de una facies caduco-espinosa de prebosque arbustivo-arborescente perteneciente a la asociación Lonicero splendidaeBerberidetum hispanicae Asensi y Rivas-Martínez 1979 (figura 6), que representa el primer estadio de sustitución en el proceso de la sucesión vegetal de la degradada clímax de bosque caduco-marcescente, la cual sólo se conserva en rodales poco extensos y altamente desestructurados en el piso supramediterráneo subhúmedo del macizo.

En el caso de las localidades P1 y P2, el estrato de prebosque viene marcado por una elevada densidad vegetal más acentuada en $\mathrm{P} 1-$, con el predominio de taxones como Prunus mahaleb L., Crataegus granatensis Boiss., Crataegus monogyna Jacq., Lonicera arborea Boiss., Amelanchier 


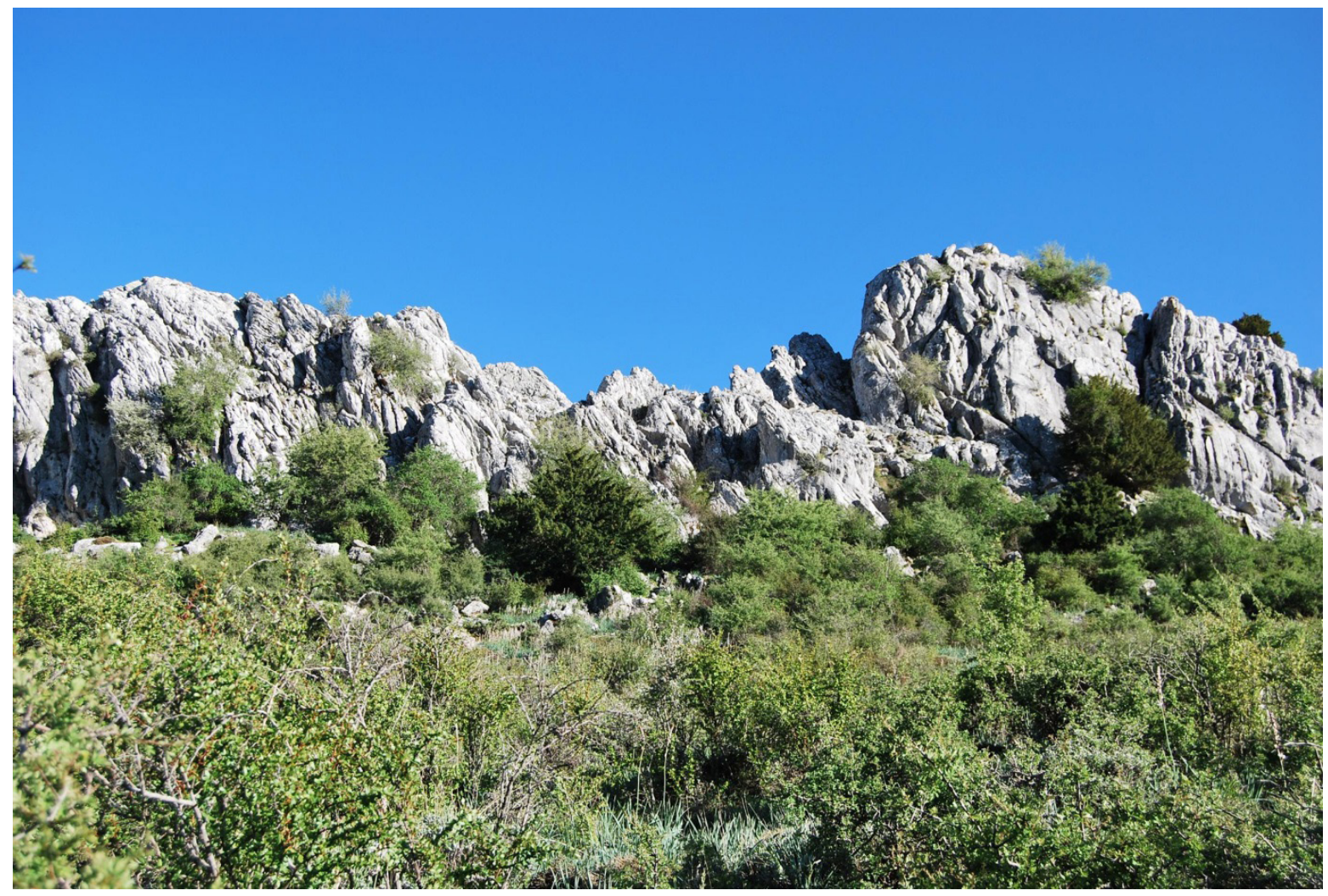

Figura 6. Estrato espinescente de Lonicero-Berberidetum hispanicae en P1 que, en la actualidad, representa el nicho ecológico de Taxus baccata en Sierra Arana.

Spinosum stratum of Lonicero-Berberidetum hispanicae in P1, which currently represents the ecological niche of Taxus baccata in Sierra Arana.

ovalis Medik., Berberis hispanica Boiss. y Reut., Rosa pimpinellifolia L., Rosa canina L., Rosa pouzinii Tratt. y Rosa sicula Tratt., siendo algo menos frecuentes otras especies como Lonicera etrusca Santi, Lonicera splendida Boiss., Cotoneaster granatensis Boiss. y Prunus ramburii Boiss. Asimismo, se conservan de forma residual taxones arbóreos integrantes de la facies climácica, como Quercus faginea ssp. faginea, Acer monspessulanus y Sorbus aria (L.) Crantz, si bien son más infrecuentes en P2 dada la mayor altitud y acusado carácter de umbría generado por la gran pendiente de esta localidad. Precisamente, el factor altitud y la orientación favorecen el refugio de elementos orófilos de tipo rastrero en ambas localidades, que indican las condiciones microclimáticas tan particulares de estos ambientes, como la sabina rastrera (Juniperus sabina L.), el enebro rastrero (Juniperus communis L. ssp. hemisphaerica (K. Presl) Nyman) y el torvisco moruno (Daphne oleoides Schreb). Cabe destacar, por último, la excepcional regeneración que están experimentado algunas de estas especies, fundamentalmente L. arborea, P. mahaleb y A. ovalis.

Matizable es el desarrollo del estrato preforestal en P3; aunque el dominio vegetal potencial donde se integra el único pie de $T$. baccata de este núcleo no varía, su ubicación en el fondo del pequeño valle que surca el arroyo Blanco (figura 7), supone un emplazamiento de ecotono entre el teórico bosque caduco-marcescente y una estrecha franja de vegetación de ribera. De este modo, además de taxones comunes con P1 y P2 (C. granatensis, C. monogyna, B. hispanica, $R$. pimpinellifolia, $R$. canina, $R$. pouzinii, $R$. sicula, A. ovalis y L. etrusca), aparecen otros claramente atribuibles a una potencial sauceda edafohigrófila -altamente desestructurada en la actualidad-, como Salix atrocinerea Brot., Rubus ulmifolius Schott., Juncus inflexus L. y Dactylorhiza elata (Poir.) Soó. Esta comunidad se integra en las saucedas béticas basófilas de Salicetum discoloro-angustifoliae Rivas-Martínez ex G. López 1976 corr. Alcaraz et al. 1991 y Crataego granatensis-Salicetum neotrichae Díez-Garretas, Asensi y Molero en Rivas-Martínez (2011).

\section{DISCUSIÓN}

Las especiales condiciones geoecológicas en las que aparece T. baccata en Sierra Arana indican su carácter relicto y seriamente amenazado. Por tanto, cabe preguntarse acerca de la futura supervivencia natural de la especie en el macizo, y si es posible actuar para favorecer su conservación y regeneración. Esta cuestión es especialmente relevante ante el peligro que representan los procesos de cambio ambiental en la Cuenca del Mediterráneo, identificada como una de las áreas más vulnerables, por el ca- 


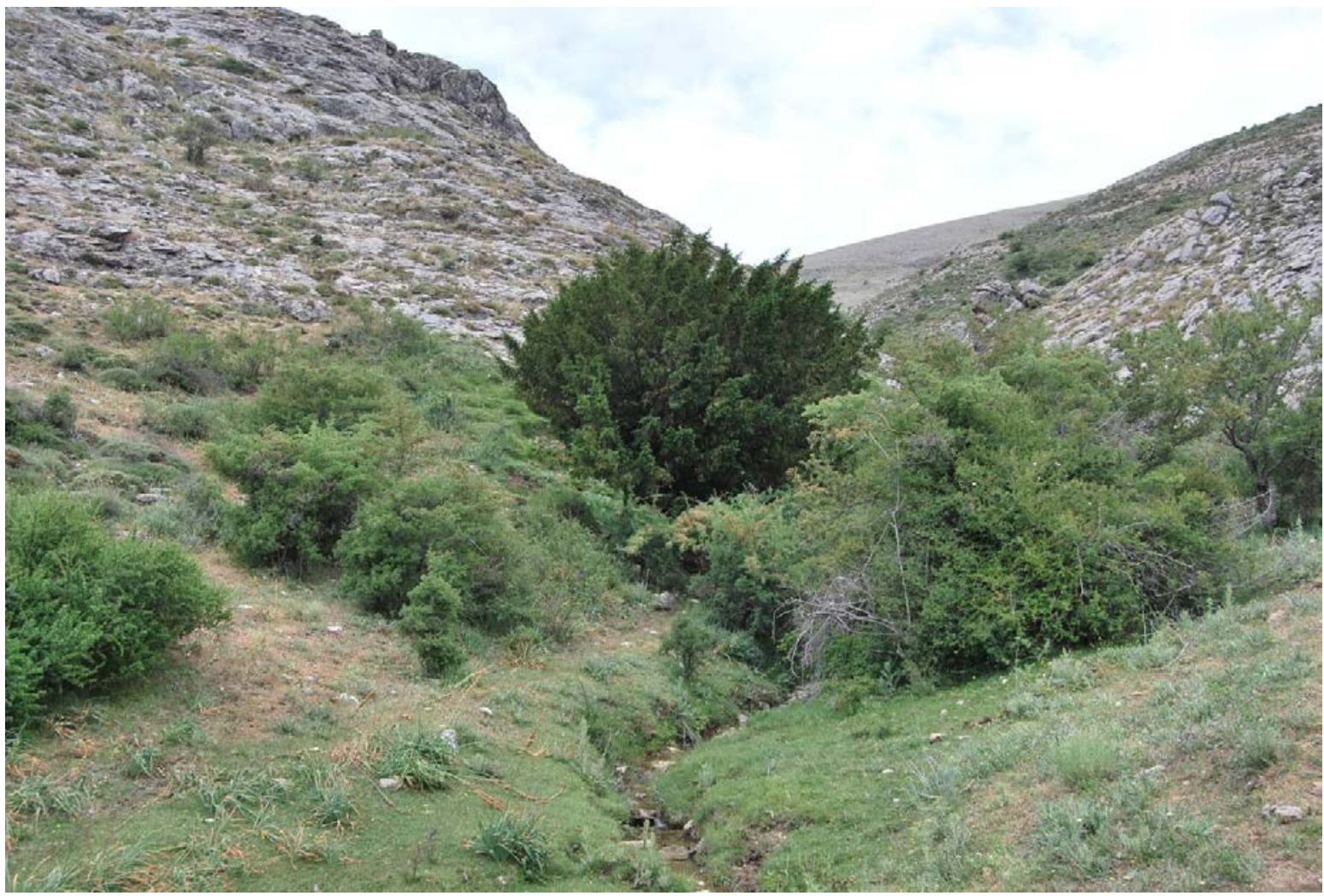

Figura 7. Hábitat y nicho ecológico del único ejemplar de Taxus baccata que compone el núcleo P3, "Arroyo Blanco". Habitat and ecological niche of the single specimen that integrates the core P3, "Arroyo Blanco".

lentamiento y desecación de su clima (Giorgi 2006). La región mediterránea europea -a la que pertenece el ámbito de estudio- se encuentra en mayor peligro al situarse en una zona de transición entre el clima árido del Norte de África y el templado-húmedo del Centro de Europa (RuizLabourdette et al. 2012).

La especie parece estar avocada a su extinción. Este fatal desenlace es debido al retroceso experimentado por los nichos ecológicos húmedos de tipo forestal. Además, su explotación efectiva como materia prima ha sido confirmada por los testimonios recabados; la dureza y resistencia de la madera de tejo para la elaboración de diversas herramientas y aparejos agrarios han favorecido un aprovechamiento tradicional mediante las denominadas "talas de recolección", actualmente inexistentes ${ }^{3}$. Ambas circunstancias han debi-

\footnotetext{
Blanco et al. (2008) resumen los aprovechamientos etnobotánicos principales de $T$. baccata en los siguientes: el empleo de su madera para usos múltiples (aparejos agrarios, juguetes, elementos de construcción, instrumentos musicales, etc.); el consumo en algunos lugares de los arilos de la semilla -única parte del tejo no tóxica-o su utilización como reclamo de caza cuando está madura en el árbol; el empleo de ramas como forraje, sobre todo para el ganado bovino; el uso medicinal tradicional (en la actualidad dentro de la farmacología científica) y veterinario de determinadas partes del tejo, como frutos, hojas, ramas y cortezas; por último, su importante valor ornamental y cultural (religioso, mítico, mágico, protector, etc.).
}

do contribuir decisivamente a la rareza de esta especie en Sierra Arana.

La problemática principal hoy día se asocia a los daños que sufre $T$. baccata por parte de los herbívoros -domésticos y salvajes-, cuya presencia habitual en estos nichos ecológicos dificulta en gran medida, incluso impide totalmente, que en la periferia y bajo los ejemplares femeninos puedan prosperar plantones a partir de las semillas caídas; en este sentido, cabe señalar que el número de semillas observado bajo los árboles es bastante escaso, lo que lleva a pensar que también existe un importante consumo de estas por parte de aves y roedores.

Junto a ello, las características demográficas de la población -bajo número de individuos, notable desequilibrio sexual, predominio de ejemplares viejos y escasez de elementos jóvenes sin plántula alguna- no solo definen una escasa capacidad de regeneración y, por tanto, de expansión de manera natural de la especie, sino su previsible extinción a medio plazo a pesar de su refugio en microambientes que mantienen condiciones favorables para el desarrollo del tejo.

Las estimaciones de la edad de T. baccata obtenidas mediante la razón de crecimiento diametral (Hulme 1996) (1 mm por año), parecen cercanas a la realidad; si bien las condiciones microclimáticas de los hábitats han permitido la preservación de la especie, éstas no favorecen 
en absoluto su crecimiento ya que el periodo vegetativo efectivo se limita a finales de la primavera y comienzos del verano; por el contrario, en hábitats más oceánicos, de menor rigor climático -tanto invernal como estival-, la tasa de crecimiento de 2,86 mm señalada por Burgarella et al. (2010) parece más acertada. Ello implicaría que los ejemplares más longevos presentasen edades superiores a 500 años, siendo supervivientes de una de las etapas históricas más agresivas con el medio vegetal en la Península Ibérica, como fue la Edad Media y el inicio de la Moderna (Muñoz-Sobrino et al. 2005, Carrión et al. 2010). En estos años se extendieron las roturaciones, amplificando la deforestación estable o incluso definitiva que se inició con las sociedades prerromanas (Ferreras y Arozena 1987).

Estas circunstancias hacen pensar que los núcleos identificados son herencia, con gran certeza, de antiguas tejedas o, al menos, de bosques de tipo húmedo donde estas eran un elemento destacado. Un desarrollo marginal que puede explicar, asimismo, por qué no se ha encontrado la especie en otros enclaves donde las condiciones ecológicas en general, y las fitocenóticas en particular, son muy similares a P1, P2 y P3; en algunos de esos lugares, la toponimia recuerda la presencia antigua del tejo (como el "cortijo de la Tejera").

En relación a la posible regeneración asistida encaminada a la conservación de T. baccata en Sierra Arana, cabe mencionar una serie de experiencias que, desde la década de 1990, se han llevado a cabo en otros ambientes montañosos de la Cordillera Bética, con características ecológicas similares a las del macizo. Estas actuaciones han demostrado que es posible la recuperación de la especie y de sus hábitats mediante su protección y, sobre todo, su reforestación. En concreto, así ha sucedido en las sierras de Tejeda, Almijara, Cazorla y de Las Nieves, donde las repoblaciones han mostrado unos niveles de éxito bastante elevados cuando se le han aplicado riegos de mantenimiento en la época más desfavorable (Costa 2006). En otros casos, como en la Sierra de Baza, las plantaciones han fracasado en su mayoría debido a la falta de humedad estival (Costa 2006), lo que demuestra que las condiciones microclimáticas locales no siempre son suficientes para la regeneración de $T$. baccata debido a la extrema sequedad del verano, incluso allí donde se repueble artificialmente. El factor riego, por tanto, es determinante para el éxito de las reforestaciones en ambientes mediterráneos, incluso fuera de la Cuenca, tal y como han experimentado Becerra et al. (2013) en zonas costeras de clima mediterráneo del centro de Chile.

Considerando ambas cuestiones, protección y reforestación, sería posible favorecer la regeneración de T. baccata en Sierra Arana. En el primer caso, es necesario implantar las siguientes medidas de resguardo directo tanto de los individuos existentes -especialmente de aquellos en peor estado de conservación o más amenazados en relación a los factores ya conocidos-, como de la fitocenosis de la que forman parte:
1. Necesidad de limitar el acceso de herbívoros a los núcleos identificados o, al menos, a aquellos ejemplares en mayor peligro, mediante recintos cercados a través de mallas metálicas.

2. Realización de controles fitosanitarios y selvícolas periódicos en los nichos ecológicos de T. baccata, con el objeto de detectar posibles amenazas en forma de enfermedades, plagas, etc., y para controlar la posible expansión de determinadas especies cuyo desarrollo puede interferir en su dinámica natural.

3. Protección específica, en todo el macizo, de la fauna que contribuye a la dispersión de sus semillas, en especial de las aves frutívoras.

4. Ampliación de los límites del Parque Natural de la Sierra de Huétor - dada su proximidad a P2 y P3- hasta abarcar todos los ejemplares de T. baccata localizados.

5. Concienciar a la sociedad respecto a la singularidad y carácter relicto y amenazado del tejo en el macizo mediante actividades divulgativas e informativas, aprovechando para ello el marco del Parque Natural y la gran afluencia de visitantes que éste recibe.

De cara a favorecer la regeneración de la especie es necesario abordar un programa de reforestación en aquellas áreas que se identifiquen como más adecuadas para ello. Considerando el marco general de actuaciones de este tipo llevadas a cabo en la Cordillera Bética, y particularizando para el caso de Sierra Arana, dicho programa debe incluir las siguientes cuestiones:

- Repoblación de T. baccata en las tres localidades donde se conserva en la actualidad.

- Reforestación en otros enclaves en los que, aunque no se ha localizado el taxón, los nichos ecológicos coinciden con los existentes en P1, P2 y P3. Para ello, se proponen siete sectores (figura 8) que presentan, en parte, conectividad entre sí, y que además incluyen las poblaciones actuales. Se procura así un escenario futuro en el que los distintos núcleos de vegetación configurarán un ecosistema poco fragmentado.

- Composición diversa en las repoblaciones en cuanto al género -individuos masculinos y femeninos- y la edad -desde plántulas hasta ejemplares de varios años-.

- Utilización, a ser posible, de ecotipos genéticamente adecuados al emplazamiento considerado. Este aspecto es importante para conseguir poblaciones futuras puras dada la variabilidad genética de $T$. baccata incluso en núcleos cercanos entre sí, como ha demostrado Hulme (1996) para distintas regiones forestales de Alemania. En este sentido, el cercano vivero para el cultivo de T. baccata en La Resinera (Sierra de Almijara), supone cubrir esta necesidad al trabajar con especies genéticamente adscritas a las montañas béticas.

- Incorporación en las repoblaciones de otros plantones y ejemplares jóvenes de especies arbóreas, arborescentes y arbustivas que caracterizan la fitocenosis potencial donde se integra T. baccata -por ejemplo A. granaten- 


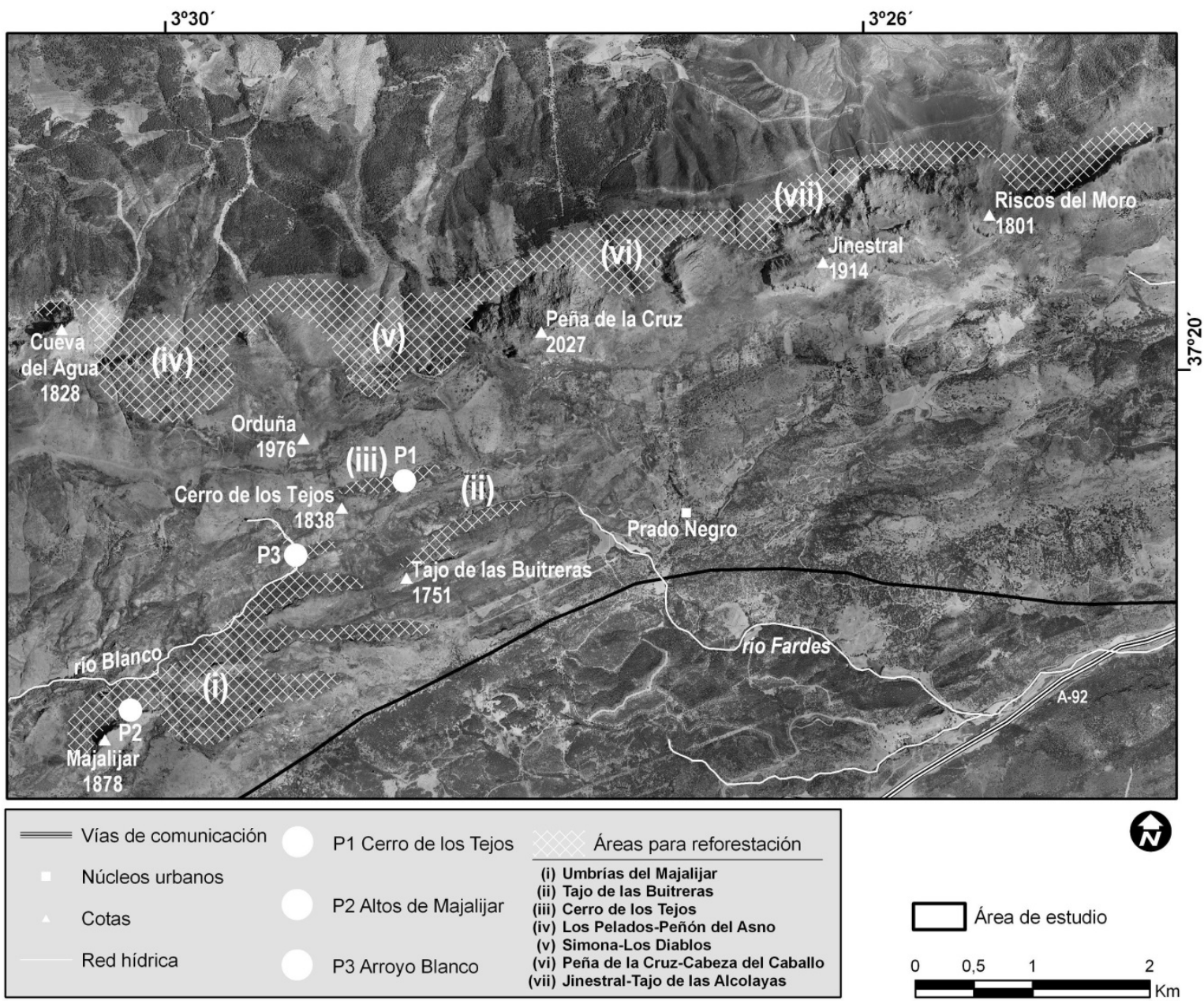

Figura 8. Zonas propuestas para la reforestación de Taxus baccata en Sierra Arana. Areas proposed for reforestation of Taxus baccata in Sierra Arana.

sis, Q. faginea, S. aria, C. monogyna, C. granatensis, P. mahaleb, L. etrusca, B. hispanica о A. ovalis-. Esta actuación favorecerá la regeneración del bosque climácico y de su estrato de preforestal; estas facies de vegetación actúan bajo condiciones ambientales mediterráneas como formación nodriza protectora de especies como T. baccata. Además, supondrá un aporte de alimentos para las aves dispersoras de semillas y contribuirá a crear unas condiciones microclimáticas a nivel del suelo que ayudan a los individuos jóvenes y plántulas a afrontar las duras y estresantes condiciones del periodo estival (García et al.1999, 2000, 2007, Hódar et al. 1998, García y Obeso 2003, Castro et al. 2004, Martínez et al. 2008, Burgarella et al. 2010).

- Protección de las zonas repobladas mediante cercados que impidan el ramoneo y el pisoteo de las plántulas e individuos jóvenes por parte de los herbívoros.

- Seguimiento exhaustivo durante las primeras fases de crecimiento de las especies plantadas para intentar asegurar su asentamiento vital, sobre todo de los taxones más susceptibles de perecer por estrés hídrico estival o periodos de sequía. Para ello, es necesario contar con una estrategia preconcebida y con los medios adecuados para poder abordar el riego de las plantaciones. Este aspecto es un punto básico para garantizar un nivel de éxito razonable en las tareas de repoblación.

- Conciliación, por último, entre los intereses privados de los propietarios de las tierras donde se asientan la mayor parte de los tejos y los de carácter público de las distintas administraciones responsables de la gestión medioambiental del territorio.

\section{CONCLUSIONES}

Los resultados de esta investigación evidencian que el actual estado de la población de T. baccata en Sierra Arana es crítico. Sus escasas posibilidades de regeneración y expansión de manera natural la sitúan al borde de la extinción; ello permite catalogar al tejo como un taxón altamente singular y de excepcional valor ecológico en este terri- 
torio. Su carácter relicto actual obedece al mantenimiento de unas condiciones ecológicas mínimamente favorables para su refugio en micro-ambientes particulares, aunque en riesgo debido al escenario macroclimático previsto; además, su conservación en el futuro no está garantizada por las propias características de la población en su conjunto, y a causa de su desarrollo en el seno de degradados bosques caduco-marcescentes intensamente castigados por la explotación antrópica del medio.

Por ello, T. baccata debe ser objeto de una gestión prioritaria. Deben conservarse los ejemplares actuales y sus nichos ecológicos mediante medidas encaminadas, básicamente, a su protección frente a la herbivoría. También debe recuperarse la especie a través de un programa de reforestación. Estas experiencias pueden ser implementadas en otras poblaciones amenazadas de distribución finícola.

Es necesario, asimismo, profundizar aún más en el conocimiento paleo-botánico de Sierra Arana para interpretar el significado ecológico actual tanto de especies de elevada singularidad, caso de T. baccata, como del conjunto de dominios vegetales que integran su paisaje vegetal. Para ello, se plantean dos líneas de investigación a desarrollar en el futuro: La revisión de las fuentes históricas y el estudio pedoantracológico.

\section{AGRADECIMIENTOS}

Los autores desean agradecer sinceramente el asesoramiento de D. Joaquín Molero Mesa, Catedrático de Botánica de la Universidad de Granada (España), en las cuestiones directamente relacionadas con la flora.

\section{REFERENCIAS}

Alcaraz F. 1996. Fitosociología integrada, paisaje y biogeografía. In Loidi J ed. Avances en Fitosociología. Bilbao, España, p. 59-94.

Becerra PI, G Cruz, S Ríos, G Castelli. 2013. Importance of irrigation and plant size in the establishment success of different native species in a degraded ecosystem of central Chile. Bosque 34(1): 103-111.

Blanca G, B Cabezudo, M Cueto, C Morales, C Salazar eds. 2011. Flora Vascular de Andalucía Oriental ( $2^{\mathrm{a}}$ edición corregida y aumentada). Sevilla, España. Consejería de Medio Ambiente - Junta de Andalucía. 1.751 p.

Blanco E, F Vasco, I Abellà, S Cortés. 2008. Tejo y cultura: de la tradición etnobotánica a la farmacología científica. In Caritat A dir, Fontaniol M coor. II Jornadas sobre el tejo en el Mediterráneo Occidental. Gerona, España, p. 11-34.

Burgarella C, E Berganzo, M Zabala, A Prada, A Iglesias, M Riba, M Mayol, G Vendramin, S González. 2010. Aspectos genéticos y demográficos de Taxus baccata en la red de Parques Nacionales. In Ramírez L, B Asensio eds. Proyectos de investigación en la red de Parques Nacionales 2007 2010. Madrid, España, p. 125-137.

Cabezudo B, AV Pérez-Latorre, F Casimiro-Soriguer, M Pavón, N Hidalgo. 2011. Contribuciones a la flora vascular de Andalucía (España). Algunas plantas interesantes de Andalu- cía oriental (Málaga-Granada). Acta Botánica Malacitana 36: 213-225.

Carrión, JS, S Fernández, P González, G Gil, E Badal, Y Carrión, L López, JA López, E Fierro, F Burjachs. 2010. Expected trends and surprises in the Lateglacial and Holocene vegetation history of the Iberian Peninsula and Balearic Islands. Review of Palaeobotany and Palynology 162 (3): 458-475.

Castro J, R Zamora, JA Hódar, JM Gómez. 2004. Seedling establishment of a boreal tree species (Pinus sylvestris) at its southernmost distribution limit: consequences of being in a marginal Mediterranean habitat. Journal of Ecology 92: 266-277.

Castroviejo S. 2010. Flora Ibérica. Plantas Vasculares de la Península Ibérica e Islas Baleares. Madrid, España. Real Jardín Botánico, Consejo Superior de Investigaciones Científicas. Volúmenes I-XXI.

Charco J. 2006. Biogeografía del tejo (Taxus baccata) en el Norte de África. In Serra L coor. I Jornadas sobre el tejo en el Mediterráneo Occidental. Valencia, España, p. 177-184.

Costa JC. 2006. Distribución y ecología de las tejedas en Andalucía: propuesta de actuaciones de conservación, regeneración y restauración. In Serra L coor. I Jornadas sobre el tejo en el Mediterráneo Occidental. Valencia, España, p. 161-170.

Ferreras C, ME Arozena. (1987). Los Bosques, Guía Física de España. Madrid, España. Alianza Editorial. 391 p.

García D, R Zamora, JA Hódar, JM Gómez. 1999. Age structure of Juniperus communis L. in the Iberian Peninsula: conservation of remnant populations in Mediterranean mountains. Biological Conservation 87: 215-220.

García D, R Zamora, JA Hódar, JM Gómez, J Castro. 2000. Yew (Taxus baccata L.) regeneration is facilitated by fleshyfruited shrubs in Mediterranean environments. Biological Conservation 95: 31-38.

García D, JR Obeso. 2003. Facilitation by herbivore-mediated nurse plants in a threatened tree, Taxus baccata: local effects and landscape level consistency. Ecography 26: 739-750.

García D, I Martínez, JR Obeso. 2007. Seed transfer among birddispersed trees and its consequences for post-dispersal seed fate. Basic and Applied Ecology 8: 533-543.

Giorgi F. 2006. Climate change hot-spots. Geophysical Research Letters 33, L08707. 4 p.

Hódar JA, J Castro, JM Gómez, D García, R Zamora. 1998. Efects of herbivory on growth and survival of seedlings and saplings of Pinus sylvestris nevadensis in SE Spain. In Papanastasis VP, D Peters eds. Ecological Basis of Livestock Grazing in Mediterranean Ecosystems. Luxemburgo, Luxemburgo. p. 264-267.

Hulme P. 1996. Natural regeneration of yew (Taxus baccata L.). Microsite, seed or herbivore limitation. Journal of Ecology 84(6): 853-861.

IGME (Instituto Geológico y Minero de España, ES). 2012. Mapa geológico de España. Instituto Geológico y Minero de España. Ministerio de Economía y Competitividad del Gobierno de España. Consultado 25 may. 2013. Disponible en http://www.igme.es

Martínez I, D García, JR Obeso. 2008. Differential seed dispersal patterns generated by a common assemblage of vertebrate frugivores in three fleshy-fruited trees. Ecoscience 15: 189199. 
MAPA (Ministerio de Agricultura, Pesca y Alimentación, ES). 1991. Mapa forestal de España. Madrid, España. Ministerio de Agricultura, Pesca y Alimentación. Hoja 5-11 (GranadaMálaga).

Muñoz-Sobrino, C, P Ramil-Rego, L Gómez-Orellana, RA Díaz. 2005. Palynological data on major Holocene climatic events in NW Iberia. Boreas 34: 381-400.

Nogués-Bravo D. 2009. Predicting the past distribution of species climatic niches. Global Ecology and Biogeography 18: 521-531.

Olmedo-Cobo JA. 2011. Análisis biogeográfico y cartografía de la vegetación de la Sierra de Baza (Provincia de Granada). El estado actual de las fitocenosis de una montaña mediterránea intensamente humaniza. Tesis Doctoral Geografía. Granada, España. Facultad de Filosofía y Letras, Universidad de Granada. 803 p.

Oyonarte C, A Pérez-Pujalte, V Aranda, T Pérez-López. 2006. Mapa digital de suelos. Provincia de Granada: Moreda-992. Dirección general para la biodiversidad, Madrid.

Pérez-Latorre AV, A Galán de Mera, B Cabezudo. 1999. Propuesta de aproximación sintaxonómica sobre las comunidades de gimnospermas de la provincia Bética (España). Acta Botánica Malacitana 24: 257-262.

Proyecto Lucdeme (1995-1998). Mapa de suelos, escala 1:100.000. Hojas: 1009, 991, 1010. Universidad de Granada, Icona, Ministerio de Agricultura, Pesca y Alimentación, Granada - Madrid.

Regato P, J Gamisans, M Gruber. 1995. A syntaxonomical study of Pinus nigra subsp. salzmannii forest in the Iberian Peninsula. Phytocoenologia 25(4): 561-578.

Rivas-Martínez S. 2008. Clasificación Bioclimática de la Tierra. Centro de Investigaciones Fitosociológicas - Universidad Complutense de Madrid. Consultado 2 jun. 2013. Disponible en http://www.globalbioclimatics.org/book/bioc/global_bioclimatics-2008_00.htm.
Rivas-Martínez S. 2011. Memoria del Mapa de Vegetación Potencial de España. Itinera Geobotánica 18: 5-800.

Rivas-Martínez S, A Asensi, B Díez-Garretas, J Molero, F Valle. 1997. Biogeographical synthesis of Andalusia (southern Spain). Journal of Biogeography 24: 915-928.

Rivas-Martínez S, J Loidi. 1999. Bioclimatology of the Iberian Peninsula. Itinera Geobotánica 13: 41-47.

Ruiz-Labourdette D, D Nogués-Bravo, HS Ollero, MF Schmitz, FD Pineda. 2012. Forest composition in Mediterranean mountains is projected to shift along the entire elevational gradient under climate change. Journal of Biogeography 39: 162-176.

Sanz R, F Pulido, D Nogués-Bravo. 2009. Predicting mechanisms across scales: amplified effects of abiotic constraints on the recruitment of yew (Taxus baccata). Ecography 32: 993-1000.

Svenning JC, E Magard. 1999. Population ecology and conservation status of the last natural population of English yew (Taxus baccata) Denmark. Biological Conservation 88: 173-182.

Thomas PA, A Polwart. 2003. Taxus baccata L. Journal of Ecology 91: 489-524.

Torres JA, E Cano, A García-Fuentes. 1996. Aportaciones al estudio fitosociológico de las comunidades de boj (Buxus sempervirens) en el sector subbético (Andalucía, España). Acta Botánica Malacitana 21: 319-321.

Valle F. 2003. Mapa de Series de Vegetación de Andalucía. Madrid, España. Consejería de Medio Ambiente - Junta de Andalucía. $132 \mathrm{p}$.

Valle F, JF Mota, F Gómez-Mercado. 1988. Datos sobre la vegetación orófila de Andalucía Oriental (España). Documents Phytosociologiques 11: 459-464.

Vidakovic M. 1991. Conifers: Morphology and Variation. Graficki Zavod Hrvatske, Zagreb, Croatia. In Thomas PA, A Polwart eds. 2003. Taxus baccata L. Journal of Ecology 91: 489-524.

Recibido: 03.07.13

Aceptado: 09.01.14 